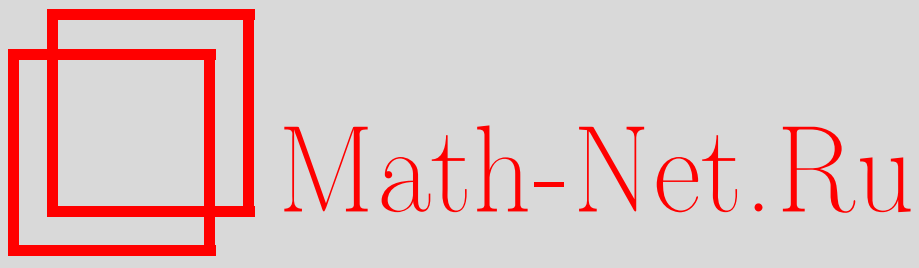

Р. В. Шамин, О пространствах начальных данных для дифференциальных уравнений в гильбертовом пространстве, Матем. сб., 2003, том 194, номер 9, 141-156

DOI: https://doi.org/10.4213/sm770

Использование Общероссийского математического портала Math-Net.Ru подразумевает, что вы прочитали и согласны с пользовательским соглашением http://www . mathnet.ru/rus/agreement

Параметры загрузки:

IP: 3.89 .185 .249

26 апреля 2023 г., $13: 18: 11$ 


\author{
Р. В. Шамин
}

\title{
О пространствах начальных данных для дифференциальных уравнений в гильбертовом пространстве
}

\begin{abstract}
Рассматриваются пространства начальных данных для дифференциальных уравненией в гильбертовом пространстве. Получены необходимые и достаточные условия сильной разрешимости параболических дифференциально-разностных уравнений и параболических функционально-дифференциальных уравнений с растяжением и сжатием аргументов.

Библиография: 21 название.
\end{abstract}

\section{§1. Введение}

При изучении задачи Коши для дифференциального уравнения в гильбертовом пространстве $H$

$$
\begin{gathered}
u^{\prime}(t)+\mathscr{A} u(t)=0, \quad t>0 \\
u(0)=\varphi \in H
\end{gathered}
$$

где оператор $-\mathscr{A}: \mathscr{D}(\mathscr{A}) \subset H \rightarrow H$ является генератором аналитической полугрупшы в пространстве $H$, возникает важный вопрос о сушествовании сильных решений задачи (1.1), (1.2) (см. [1], [2]). В случае, когда оператор $\mathscr{A}$ является неограниченным, хорошо известно, что не для всех $\varphi \in H$ сушествует сильное решение задачи (1.1), (1.2) (см. [1]). Множество всех $\varphi \in H$, для которых существует сильное решение, называется пространством начальных данных задачи (1.1), (1.2).

Изучение пространств начальных данных тесно связано с известной проблемой Като. В [3] Т. Като сформулировал следуюшую проблему: совпадает ли область определения $\mathscr{D}\left(\mathscr{A}^{1 / 2}\right)$ с $\mathscr{D}\left(\left(\mathscr{A}^{*}\right)^{1 / 2}\right)$ ? В [4] показано, что, вообще говоря, ответ отрицательный. После этого проблема Като была переформулирована для эллиптических операторов с измеримыми коэффициентами (см. [5] и библиографию там же).

В настоящей работе выделен широкий класс коэрцитивных операторов $\mathscr{A}$, для которых имеет место равенство $\mathscr{D}\left(\mathscr{A}^{1 / 2}\right)=\mathscr{D}\left(\left(\mathscr{A}^{*}\right)^{1 / 2}\right)$. Этот класс включает, в частности, дифференциально-разностные операторы, функционально-дифференциальные операторы с растяжением и сжатием аргументов и другие. Кстати, отметим, что функционально-дифференциальньми уравнениями также занимались Т. Като и Дж. Б. Маклеод [6].

Работа выполнена при финансовой поддержке Российского фонда фундаментальных исследований (грант № 01-01-01030) и фонда INTAS (грант № 97-30551).

$$
\text { (C) Р. В. ШАмин } 2003
$$


Статья состоит из пяти параграфов. Во втором и третьем параграфах мы приводим постановку задачи и определяем пространство начальных данных. Основной результат об интерполяции областей определения коэрцитивных операторов получен в $\S 4$. В $\S 5$ мы применяем абстрактные результаты к параболическим задачам, в частности функционально-дифференциальньм, и приводим различные примеры.

Параболические функционально-дифференциальные уравнения изучались в [7], [8]. В работе [9] показано, что область определения дифференциально-разностных операторов может содержать негладкие функции, не принадлежащие пространствам Соболева. С этим связаны большие трудности, возникающие при исследовании пространств начальных данных соответствующих параболических задач. При дополнительных условиях на область $Q_{T}$, в которой рассматриваются уравнения, и на область определения эллиптического дифференциально-разностного оператора пространство начальных данных для параболических дифференциально-разностных уравнений исследовано в [10]. Подход, предлагаемый в данной работе, позволяет получить описание пространств начальных данных для различных классов параболических функционально-дифференциальных уравнений без дополнительных ограничений на область $Q_{T}$ и область определения $\mathscr{D}(\mathscr{A})$.

Краткое изложение основных результатов было дано в [11].

\section{§ 2. Постановка задачи}

Пусть $V$ и $H$ - сепарабельные гильбертовы пространства, и пусть $V$ плотно и непрерывно вложено в $H$. Отождествим пространство $H$ с его сопряженным, тогда мы получим $V \subset H \subset V^{\prime}$, где каждое пространство плотно в последующем.

Рассмотрим непрерывный оператор А: $V \rightarrow V^{\prime}$.

ОПРЕДЕЛЕНИЕ 2.1. Оператор А называется $V$-коэриитивным, если для любого $v \in V$

$$
\operatorname{Re}\langle\mathrm{A} v, v\rangle \geqslant c_{1}\|v\|_{V}^{2}
$$

где $c_{1}>0$ не зависит от $v$.

В дальнейшем будем предполагать, что оператор А является $V$-коэрцитивным.

ЗАмЕчАниЕ 2.1. В силу теоремы 9.1 [12; гл. 2] оператор А взаимно однозначно отображает $V$ на $V^{\prime}$.

Рассмотрим полуторалинейную форму $a[u, v]$ в $H$, определенную по формуле $a[u, v]=\langle\mathrm{A} u, v\rangle, \mathscr{D}(a)=V$. В силу $(2.1)$ форма $a$ является замкнутой секториальной формой в $H$. Действительно, для произвольного $u \in V$ мы имеем

$$
|\operatorname{Im} a[u, u]| \leqslant|a[u, u]| \leqslant c_{2}\|u\|_{V}^{2}
$$

Из последнего неравенства и из (2.1) следует, что числовая область значений $\Theta(a)$ лежит в секторе

$$
\Theta(a) \subset\left\{\lambda \in \mathbb{C}:-\theta_{1}<\arg \lambda<\theta_{1}\right\}
$$

где $\theta_{1}=\operatorname{arctg}\left(c_{2} / c_{1}\right)<\pi / 2$.

В силу теоремы о представлении 2.1 [13; гл. 6] существует замкнутый, плотно определенный, секториальный оператор $\mathscr{A}: \mathscr{D}(\mathscr{A}) \subset H \rightarrow H$, порожденный формой $a$, такой, что $(\mathscr{A} u, v)_{H}=a[u, v], u \in \mathscr{D}(\mathscr{A}), v \in V$. Очевидно, $\mathscr{A} u=\mathrm{A} u$, когда $u \in \mathscr{D}(\mathscr{A})=\{u \in V: \mathrm{A} u \in H\}$. 
Будем рассматривать область определения $\mathscr{D}(\mathscr{A})$ как гильбертово пространство со скалярным произведением

$$
(u, v)_{\mathscr{D}(\mathscr{A})}=(\mathscr{A} u, \mathscr{A} v)_{H}+(u, v)_{H}
$$

Пусть $Y$ - гильбертово пространство. Через $L_{2}(0, T ; Y)$ обозначим гильбертово пространство измеримых функций со значениями в $Y$ с конечной нормой

$$
\|u\|_{L_{2}(0, T ; Y)}=\left(\int_{0}^{T}\|u(t)\|_{Y}^{2} d t\right)^{1 / 2}
$$

Введем гильбертово пространство $\mathscr{W}(\mathscr{A})=\left\{w \in L_{2}(0, T ; \mathscr{D}(\mathscr{A})): w^{\prime} \in L_{2}(0, T ; H)\right\}$ со скалярным произведением

$$
(u, v)_{\mathscr{W}(\mathscr{A})}=\int_{0}^{T}\left(u^{\prime}, v^{\prime}\right)_{H} d t+\int_{0}^{T}(\mathscr{A} u, \mathscr{A} v)_{H} d t+\int_{0}^{T}(u, v)_{H} d t
$$

где производные по $t$ понимаются в смысле распределений со значениями в $L_{2}(0, T ; H)$.

ЗАмечАниЕ 2.2. В силу теоремы 3.1 [12; гл. 1] функция $w \in \mathscr{W}(\mathscr{A})$ имеет след $\left.w\right|_{t=0} \in H$.

Будем рассматривать диффференциальное уравнение в гильбертовом пространстве $H$

$$
u^{\prime}(t)+\mathscr{A} u(t)=f(t), \quad t \in(0, T),
$$

с начальным условием

$$
\left.u\right|_{t=0}=\varphi
$$

где $0<T<\infty, f \in L_{2}(0, T ; H)$ и $\varphi \in H$.

ОПРЕДЕЛЕНИЕ 2.2. Функция $u \in \mathscr{W}(\mathscr{A})$ называется сильным решением задачи (2.3), (2.4), если $и$ удовлетворяет (2.3) при почти всех $t \in(0, T)$ и (2.4) в смысле замечания 2.2 .

\section{§3. Сильная разрешимость}

Для исследования сильной разрешимости задачи $(2.3),(2.4)$ воспользуемся методами теории полугрупп.

ОПРЕДЕЛЕНИЕ 3.1. Пусть $X$ - банахово пространство. Однопараметрическое семейство линейных ограниченных операторов $T_{t}: X \rightarrow X, t \geqslant 0$, назьвается сильно непрерывной полугруппой или $C_{0}$-полугруппой, если

1) $T_{0}=I$

2) $T_{t+s}=T_{t} T_{s}, t, s \geqslant 0$

3) $\lim _{t \searrow 0} T_{t} x=x \forall x \in X$.

ОПРЕДЕЛЕНИЕ 3.2. Полугруппа класса $C_{0}$ назьвается сжсимающей, если $\left\|T_{t}\right\| \leqslant 1, t \geqslant 0$. 
ОПРЕДЕЛЕНИЕ 3.3. Линейньй оператор $\mathrm{G}: X \rightarrow X$, определенньй по формуле

$$
\mathrm{G} x=\lim _{t \searrow 0} \frac{T_{t} x-x}{t}, \quad x \in D(\mathrm{G})=\left\{x \in X: \lim _{t \searrow 0} \frac{T_{t} x-x}{t} \text { сушествует }\right\},
$$

называется генератором сильно непрерывной полугруппь $\left\{T_{t}\right\}$.

Обозначим $\Delta=\left\{z \in \mathbb{C}: \varphi_{1}<\arg z<\varphi_{2}\right\}$, где $\varphi_{1}<0<\varphi_{2}$.

ОПРЕДЕЛЕНИЕ 3.4. Однопараметрическое семейство линейных ограниченных операторов $T_{z}: X \rightarrow X, z \in \Delta$, назьвается аналитической полугруппой в $\Delta$, если

1) функция $z \mapsto T_{z}$ является аналитической в $\Delta$;

2) $T_{0}=I$ и $\lim _{z \rightarrow 0, z \in \Delta} T_{z} x=x$ для любого $x \in X$;

3) $T_{z_{1}+z_{2}}=T_{z_{1}} T_{z_{2}}$ для любых $z_{1}, z_{2} \in \Delta$.

Полугруппа $T_{t}, t \geqslant 0$, называется аналитической, если ее можно продолжить до аналитической в некотором секторе $\Delta$.

Лемма 3.1. Пусть оператор А является $V$-коэрцитивныцм, тогда оператор -A является генератором аналитической полугруппь $\left\{T_{t}\right\}_{t \geqslant 0}$.

ДоказАтельство. Оператор $\mathscr{A}$ является замкнутым, плотно определенным, секториальным оператором, поэтому в силу теоремы 1.24 [13; гл. 9] оператор $-\mathscr{A}$ является инфинитезимальным производящим оператором аналитической полугрупшы $T_{t}$ в $H$.

Предположим сначала, что $f=0$. Хорошо известно, что если $\varphi \in \mathscr{D}(\mathscr{A})$, то функция $u(t)=T_{t} \varphi$ является сильным решением задачи (2.3), (2.4), где $f=0$ (см. [2]). Если оператор $\mathscr{A}$ является неограниченным, то не для всех $\varphi \in H$ будет сушествовать сильное решение задачи (2.3), (2.4). С другой стороны, условие принадлежности $\varphi \in \mathscr{D}(\mathscr{A})$ не является необходимым условием сильной разрешимости.

Введем множество $\Phi=\{\varphi \in H$ : существует сильное решение задачи (2.3), $(2.4)\}$. Ввиду линейности задачи множество $\Phi$ является линейным пространством.

ОПРЕДЕЛЕНИЕ 3.5. Пространство Ф называется пространством начальных данных для задачи (2.3), (2.4).

ЗАмечание 3.1. Если задача (2.3), (2.4) имеет сильное решение, то это решение единственно [2].

Имеют место следуюшие вложения: $\mathscr{D}(\mathscr{A}) \subset \Phi \subset H$. Для описания пространства начальных данных мы будем применять методы теории интерполяции гильбертовых пространств.

Рассмотрим гильбертово пространство $H_{1}$, плотно и непрерывно вложенное в $H$. Определим в пространстве $H$ замкнутую, симметричную, положительную форму $t[u, v]=(u, v)_{H_{1}}, \mathscr{D}(t)=H_{1}$. Пусть $T$ - ассоциированный с ней самосопряженный положительный оператор. Тогда оператор $\Lambda=T^{1 / 2}$ есть также положительный оператор в $H$ и в силу теоремы 2.23 [13; гл. 6] область определения $\mathscr{D}(\Lambda)=\mathscr{D}(t)=H_{1}$, причем $t[u, v]=(\Lambda u, \Lambda v)_{H}$.

Определим теперь интерполяционные пространства $\left[H_{1} ; H\right]_{\theta}, 0 \leqslant \theta \leqslant 1$, следуюшим образом. Положим $\left[H_{1} ; H\right]_{\theta}=\mathscr{D}\left(\Lambda^{\theta}\right), 0 \leqslant \theta \leqslant 1$, где дробная степень $\Lambda^{\theta}$ определена для положительного оператора $\Lambda$. Пространство $\left[H_{1} ; H\right]_{\theta}$ есть гильбертово пространство со скалярным произведением

$$
(u, v)_{\left[H_{1} ; H\right]_{\theta}}=\left(\Lambda^{\theta} u, \Lambda^{\theta} v\right)_{H}+(u, v)_{H} .
$$


Tеорема 3.1. Пусть оператор А есть V-коэриитивный оператор, тогда задача (2.3), (2.4) имеет единственное сильное решение для любого $f \in$ $L_{2}(0, T ; H)$ тогда и только тогда, когда $\varphi \in[\mathscr{D}(\mathscr{A}) ; H]_{1 / 2}$. Более того, это решение представляется формулой

$$
u(t)=T_{t} \varphi+\int_{0}^{t} T_{t-s} f(s) d s
$$

где $\left\{T_{t}\right\}_{t \geqslant 0}$ - аналитическая полугруппа, порожденная оператором - $\mathcal{A}$.

ДокАЗАтЕЛьство. Согласно лемме 3.1 оператор - $\mathscr{A}$ является генератором аналитической полугруппы. В силу теоремы 3.7 [1; гл. 1], которая утверждает, что функция $u_{1}(t)=\int_{0}^{t} T_{t-s} f(s) d s$ является сильным решением задачи $(2.3),(2.4)$ при $\varphi=0$, задача $(2.3),(2.4)$ имеет единственное сильное решение тогда и только тогда, когда выполнено неравенство

$$
\int_{0}^{T}\left\|\mathscr{A} T_{t} \varphi\right\|_{H}^{2} d t<\infty
$$

При этом верна формула (3.1). В силу теоремы 1.14 .5 [14; гл. 1] последнее неравенство эквивалентно включению $\varphi \in[\mathscr{D}(\mathscr{A}) ; H]_{1 / 2}$.

Из теоремы 3.1 ясно, что для решения вопроса о сильной разрешимости задачи $(2.3),(2.4)$ необходимо иметь описание пространства начальных данных $\Phi=$ $[\mathscr{D}(\mathscr{A}) ; H]_{1 / 2}$. В конкретных примерах часто возникают следующие трудности: во-первых, область определения $\mathscr{D}(\mathscr{A})$ задана неконструктивно и может быть очень сложной, во-вторых, сама операция интерполирования конкретных пространств может оказаться затруднительной.

В следуюшем параграфе мы покажем, что при выполнении некоторых естественных условий можно показать, что пространство начальных данных $\Phi$ совпадает с пространством $V$.

\section{§4. Интерполяция области определения коэрцитивного оператора}

Рассмотрим в $H$ форму $\bar{a}$, сопряженную к форме $a$. Форма $\bar{a}$ определяется по формуле $\bar{a}[u, v]=\left\langle\mathrm{A}^{*} u, v\right\rangle$, где $\mathrm{A}^{*}$ - сопряженный к А оператор. В силу теормы 2.5 [13; гл. 6] форма $\bar{a}$ порождает оператор $\mathscr{A}^{*}$, сопряженный к оператору $\mathscr{A}$. Область определения $\mathscr{D}\left(\mathscr{A}^{*}\right)$ будем рассматривать как гильбертово пространство со скалярным произведением, аналогичным (2.2).

ТЕОРема 4.1. Пусть оператор А является $V$-коэриитивным. Предположим, что имеют место непрерьвные вложсения $V \subset[\mathscr{D}(\mathscr{A}) ; H]_{1 / 2} u$ $V \subset\left[\mathscr{D}\left(\mathscr{A}^{*}\right) ; H\right]_{1 / 2}$.

Тогда $V=[\mathscr{D}(\mathscr{A}) ; H]_{1 / 2}=\left[\mathscr{D}\left(\mathscr{A}^{*}\right) ; H\right]_{1 / 2}$.

ДокАЗАТЕльство. Для произвольного $u \in H$ форма $(\mathscr{A} w, u)_{H}$ определяет линейньй непрерывный функционал $f$ на $\mathscr{D}(\mathscr{A})$ по формуле $\langle w, f\rangle=(\mathscr{A} w, u)_{H}$. Действительно,

$$
\sup _{w \in \mathscr{D}(\mathscr{A})} \frac{\left|(\mathscr{A} w, u)_{H}\right|}{\|w\|_{\mathscr{D}(\mathscr{A})}} \leqslant \sup _{w \in \mathscr{D}(\mathscr{A})} \frac{\|\mathscr{A} w\|_{H}\|u\|_{H}}{\left(\|\mathscr{A} w\|_{H}^{2}+\|w\|_{H}^{2}\right)^{1 / 2}} \leqslant\|u\|_{H} .
$$


Функционал $f$ можно представить в виде $f=\mathrm{A}_{0}^{\prime} u$, где оператор $\mathrm{A}_{0}^{\prime}$ ограничен как оператор

$$
\mathrm{A}_{0}^{\prime}: H \rightarrow(\mathscr{D}(\mathscr{A}))^{\prime}
$$

поскольку $\|f\|_{(\mathscr{D}(\mathscr{A}))^{\prime}} \leqslant\|u\|_{H}$.

Покажем, что $\mathscr{A}^{*} \subset \mathrm{A}_{0}^{\prime}$, т.е. $\mathrm{A}_{0}^{\prime} u=\mathscr{A}^{*} u$ для $u \in \mathscr{D}\left(\mathscr{A}^{*}\right)$. Пусть $u \in \mathscr{D}\left(\mathscr{A}^{*}\right)$ и $w \in \mathscr{D}(\mathscr{A})$. Обозначим $\mathrm{A}_{0}^{\prime} u=f_{1}$ и $\mathscr{A}^{*} u=f_{2}$. Тогда

$$
\left\langle w, f_{1}\right\rangle=(\mathscr{A} w, u)_{H}=\left(w, \mathscr{A}^{*} u\right)_{H}=\left\langle w, f_{2}\right\rangle .
$$

В силу произвольности $w \in \mathscr{D}(\mathscr{A})$ мы имеем $\mathscr{A}^{*} u=\mathrm{A}_{0}^{\prime} u$ в $(\mathscr{D}(\mathscr{A}))^{\prime}$, но $\mathscr{A}^{*} u \in H$ и $H \subset\left(\mathscr{D}\left(\mathrm{A}^{*}\right)\right)^{\prime}$, следовательно, $\mathscr{A}^{*} u=\mathrm{A}_{0}^{\prime} u \in H$ для $u \in \mathscr{D}\left(\mathscr{A}^{*}\right)$. Поскольку оператор $\mathscr{A}^{*}$ ограниченно отображает $\mathscr{D}\left(\mathscr{A}^{*}\right)$ в $H$, то и оператор $\mathrm{A}_{0}^{\prime}$ ограничен как оператор

$$
\mathrm{A}_{0}^{\prime}: \mathscr{D}\left(\mathscr{A}^{*}\right) \rightarrow H
$$

Из (4.1) и (4.2) в силу интерполяционной теоремы [12; гл. 1 , теорема 5.1] оператор $\mathrm{A}_{0}^{\prime}$ ограничен как оператор

$$
\mathrm{A}_{0}^{\prime}:\left[\mathscr{D}\left(\mathscr{A}^{*}\right) ; H\right]_{1 / 2} \rightarrow\left[H ;(\mathscr{D}(\mathscr{A}))^{\prime}\right]_{1 / 2} .
$$

Однако согласно теореме 6.2 о транспонировании [ $[12 ;$ гл. 1] справедливо равенство $\left[H ;(\mathscr{D}(\mathscr{A}))^{\prime}\right]_{1 / 2}=\left([\mathscr{D}(\mathscr{A}) ; H]_{1 / 2}\right)^{\prime}$. Поэтому ограничен оператор

$$
\mathrm{A}_{0}^{\prime}:\left[\mathscr{D}\left(\mathscr{A}^{*}\right) ; H\right]_{1 / 2} \rightarrow\left([\mathscr{D}(\mathscr{A}) ; H]_{1 / 2}\right)^{\prime} .
$$

Покажем, что $\mathrm{A}_{0}^{\prime} u=\mathrm{A}^{*} u$, если $u \in V$. Возьмем $u \in V$ и $w \in \mathscr{D}(\mathscr{A})$. Пусть $\mathrm{A}_{0}^{\prime} u=f_{1}$ и $\mathrm{A}^{*} u=f_{2}$. Тогда

$$
\left\langle w, f_{1}\right\rangle=(\mathscr{A} w, u)_{H}=\langle\mathrm{A} w, u\rangle=\left\langle w, \mathrm{~A}^{*} u\right\rangle=\left\langle w, f_{2}\right\rangle .
$$

$\mathrm{B}$ силу произвольности $w \in \mathscr{D}(\mathscr{A})$ мы получаем равенство $\mathrm{A}^{*} u=\mathrm{A}_{0}^{\prime} u$ в $(\mathscr{D}(\mathscr{A}))^{\prime}$, но $\mathrm{A}^{*} u \in V^{\prime}$ и $V^{\prime} \subset(\mathscr{D}(\mathscr{A}))^{\prime}$. Следовательно, $\mathrm{A}^{*} u=\mathrm{A}_{0}^{\prime} u \in V^{\prime}$.

Возьмем произвольное $f \in V^{\prime}$. Тогда $u=\left(\mathrm{A}^{*}\right)^{-1} f \in V$ и

$$
\|u\|_{V} \leqslant c_{1}\|f\|_{V^{\prime}}
$$

где $c_{1}>0$ не зависит от $f$. По предположению теоремы $u \in\left[\mathscr{D}\left(\mathscr{A}^{*}\right) ; H\right]_{1 / 2}$ и

$$
\|u\|_{\left[\mathscr{D}\left(\mathscr{A}^{*}\right) ; H\right]_{1 / 2}} \leqslant c_{2}\|u\|_{V}
$$

где $c_{2}>0$ не зависит от $f$.

В силу (4.3) получаем $\mathrm{A}_{0}^{\prime} u=f \in\left([\mathscr{D}(\mathscr{A}) ; H]_{1 / 2}\right)^{\prime}$. Учитьвая $(4.4),(4.5)$, получаем

$$
\|f\|_{\left([\mathscr{D}(\mathscr{A}) ; H]_{1 / 2}\right)^{\prime}} \leqslant c_{3}\|u\|_{\left[\mathscr{D}\left(\mathscr{A}^{*}\right) ; H\right]_{1 / 2}} \leqslant c_{3} c_{2}\|u\|_{V} \leqslant c_{3} c_{2} c_{1}\|f\|_{V^{\prime}}
$$

В силу произвольности $f \in V^{\prime}$ имеем, что $V^{\prime} \subset\left([\mathscr{D}(\mathscr{A}) ; H]_{1 / 2}\right)^{\prime}$. Переходя к сопряженным пространствам, получаем, что $[\mathscr{D}(\mathscr{A}) ; H]_{1 / 2} \subset V$. Вместе с предположением теоремы это означает, что $V=[\mathscr{D}(\mathscr{A}) ; H]_{1 / 2}$ с точностью до эквивалентности норм.

Равенство $V=\left[\mathscr{D}\left(\mathscr{A}^{*}\right) ; H\right]_{1 / 2}$ устанавливается аналогично.

Поскольку оператор $-\mathscr{A}$ является генератором аналитической полугрупшы, то для оператора $\mathscr{A}$ можно определить дробные степени $\mathscr{A}^{\theta}, 0 \leqslant \theta \leqslant 1$ (см. [3], [15]). Аналогично дробные степени определены и для $\mathscr{A}^{*}$. 
ТЕОРемА 4.2. Пусть выполнены все условия теоремы 4.1.

Тогда $\mathscr{D}\left(\mathscr{A}^{1 / 2}\right)=\mathscr{D}\left(\left(\mathscr{A}^{*}\right)^{1 / 2}\right)=V$.

ДокаЗАТЕльство. Возьмем произвольное число $\alpha \in\left(0, \frac{1}{2}\right)$. В силу теоремы 1.15 .2 [14; гл. 1] оператор $\mathscr{A}^{\alpha}$ ограниченно и взаимно однозначно отображает пространство $[\mathscr{D}(\mathscr{A}) ; H]_{1 / 2}$ на пространство $[\mathscr{D}(\mathscr{A}) ; H]_{1 / 2-\alpha}$. Согласно теореме 4.1 имеем ограниченный оператор

$$
\mathscr{A}^{\alpha}: V \rightarrow[\mathscr{D}(\mathscr{A}) ; H]_{1 / 2-\alpha}
$$

Для любого $\alpha \in\left(0, \frac{1}{2}\right)$ введем оператор $K_{\alpha}=\Lambda^{1 / 2-\alpha} \mathscr{A}^{\alpha}$, где $\Lambda$ - положительный оператор, с помощью которого определены интерполяционные пространства $[\mathscr{D}(\mathscr{A}) ; H]_{\theta}$. Из (4.6) следует, что операторы $K_{\alpha}: V \rightarrow H$ ограничены для $\alpha \in\left(0, \frac{1}{2}\right)$.

Для произвольного фиксированного $\varphi \in \mathscr{D}(\mathscr{A})$ можно перейти к пределу в $H$

$$
\lim _{\alpha \rightarrow 1 / 2} K_{\alpha} \varphi=\lim _{\alpha \rightarrow 1 / 2} \Lambda^{1 / 2-\alpha} \mathscr{A}^{\alpha} \varphi=\lim _{\alpha \rightarrow 1 / 2} \Lambda^{1 / 2} \Lambda^{-\alpha} \mathscr{A}^{\alpha-1 / 2} \mathscr{A}^{1 / 2} \varphi=\mathscr{A}^{1 / 2} \varphi
$$

В (4.7) мы пользуемся теоремой 14.1 [15; гл. 4] об аналитичности семейства операторов $\mathscr{A}^{-t}$ при $t \geqslant 0$. Поскольку $\mathscr{D}(\mathscr{A})$ плотно в $V$, то по теореме Банаха-Штейнгауза

$$
\mathscr{A}^{1 / 2}: V \rightarrow H-\text { ограниченный оператор. }
$$

Следовательно, имеем $V \subset \mathscr{D}\left(\mathscr{A}^{1 / 2}\right)$. Покажем, что имеет место и обратное вложение. Из (4.6) следует, что операторы $K_{\alpha}^{-1}=\mathscr{A}^{-\alpha} \Lambda^{-(1 / 2-\alpha)}: H \rightarrow V$ ограничены. Для любого $\varphi \in \mathscr{D}(\mathscr{A})$ покажем, что

$$
\lim _{\alpha \rightarrow 1 / 2} K_{\alpha}^{-1} \varphi=\mathscr{A}^{-1 / 2} \varphi
$$

в $\mathscr{D}(\mathscr{A})$. Сначала покажем, что $\Lambda^{-(1 / 2-\alpha)} \varphi$ сходится к $\varphi$ в $\mathscr{D}(\Lambda)=\mathscr{D}(\mathscr{A})$ при $\alpha \rightarrow \frac{1}{2}$. Имеем

$$
\begin{aligned}
& \lim _{\alpha \rightarrow 1 / 2}\left\|\Lambda^{-(1 / 2-\alpha)} \varphi-\varphi\right\|_{\mathscr{D}(\Lambda)}=\lim _{\alpha \rightarrow 1 / 2}\left\|\Lambda\left(\Lambda^{-(1 / 2-\alpha)} \varphi-\varphi\right)\right\|_{H} \\
& \quad+\lim _{\alpha \rightarrow 1 / 2}\left\|\Lambda^{-(1 / 2-\alpha)} \varphi-\varphi\right\|_{H}=\lim _{\alpha \rightarrow 1 / 2}\left\|\Lambda^{-(1 / 2-\alpha)} \psi_{1}-\psi_{1}\right\|_{H}=0
\end{aligned}
$$

где $\Lambda \varphi=\psi_{1} \in H$. Покажем теперь, что $\mathscr{A}^{-\alpha}$ сходится к $\mathscr{A}^{-1 / 2}$ в $\mathscr{D}(\mathscr{A})$ при $\alpha \rightarrow \frac{1}{2}$. Имеем

$$
\begin{aligned}
\lim _{\alpha \rightarrow 1 / 2}\left\|\mathscr{A}^{-\alpha} \varphi-\mathscr{A}^{-1 / 2} \varphi\right\|_{\mathscr{D}(\mathscr{A})} & =\lim _{\alpha \rightarrow 1 / 2}\left\|\mathscr{A}\left(\mathscr{A}^{-\alpha} \varphi-\mathscr{A}^{-1 / 2} \varphi\right)\right\|_{H} \\
\quad+\lim _{\alpha \rightarrow 1 / 2}\left\|\mathscr{A}^{-\alpha} \varphi-\mathscr{A}^{-1 / 2} \varphi\right\|_{H} & =\lim _{\alpha \rightarrow 1 / 2}\left\|\mathscr{A}^{-\alpha} \psi_{2}-\mathscr{A}^{-1 / 2} \psi_{2}\right\|_{H}=0
\end{aligned}
$$

где $\mathscr{A} \varphi=\psi_{2} \in H$. Следовательно, имеет место (4.8). Поскольку $\mathscr{D}(\mathscr{A})$ плотно в $V$, то по теореме Банаха-Штейнгауза оператор $\mathscr{A}^{-1 / 2}: H \rightarrow V$ ограничен и имеет место вложение $\mathscr{D}\left(\mathscr{A}^{1 / 2}\right) \subset V$. Мы доказали, что $\mathscr{D}\left(\mathscr{A}^{1 / 2}\right)=V$. Поскольку оператор $\mathscr{A}^{*}$ обладает теми же свойствами, что и оператор $\mathscr{A}$, то аналогично мы имеем $\mathscr{D}\left(\left(\mathscr{A}^{*}\right)^{1 / 2}\right)=V$.

Теорема 4.2 дает положительное решение проблемы Като для класса операторов, удовлетворяющих условиям теоремы 4.1. В следующем параграфе мы рассмотрим конкретные операторы из этого класса. 


\section{§5. Пространства начальных данных для параболических задач}

В настоящем параграфе мы рассмотрим примеры различных параболических задач, для которых можно получить необходимые и достаточные условия сильной разрешимости, используя результаты предыдущего параграфа.

Пусть $\mathscr{A}$ - оператор, определенный в $\S 2$ по $V$-коэрцитивному оператору А. Рассмотрим ограниченный оператор $C: V \rightarrow H$. Будем предполагать, что оператор $C$ является ограниченньм относительно оператора $\mathscr{A}$ с относительной границей 0 , т.е. нижняя грань всех возможных констант $b$ в неравенстве

$$
\|C u\|_{H} \leqslant a\|u\|_{H}+b\|\mathscr{A} u\|_{H}, \quad u \in \mathscr{D}(\mathscr{A}),
$$

равна нулю.

Введем замкнутьй оператор $\mathscr{L}: \mathscr{D}(\mathscr{L}) \subset H \rightarrow H$, действуюший по формуле $\mathscr{L} u=\mathscr{A} u+C u, u \in \mathscr{D}(\mathscr{L})=\mathscr{D}(\mathscr{A})$.

Рассмотрим эволюционное уравнение

$$
u^{\prime}(t)+\mathscr{L} u(t)=f(t), \quad t \in(0, T),
$$

с начальным условием

$$
u(0)=\varphi \text {. }
$$

ОПРЕДЕЛЕНИЕ 5.1. Функция $u \in \mathscr{W}(\mathscr{L})$ называется сильным решением задачи (5.1), (5.2), если функция $u$ удовлетворяет (5.1) при почти всех $t \in(0, T)$ и (5.2).

Результаты предыдуших параграфов для задачи (5.1), (5.2) позволяют доказать следующий результат.

ТЕОрема 5.1. Предполохим, что выполнены условия теоремы 4.1.

Тогда для любой $f \in L_{2}(0, T ; H)$ задача (5.1), (5.2) имеет единственное сильное решение тогда и только тогда, когда $\varphi \in V$. Более того, это решение представляется формулой

$$
u(t)=T_{t} \varphi+\int_{0}^{t} T_{t-s} f(s) d s
$$

әде $\left\{T_{t}\right\}_{t \geqslant 0}$ - аналитическая полугруппа, порожденная оператором - $\mathscr{L}$.

ДокАЗАтЕльство. Сначала покажем, что оператор $-\mathscr{L}$ порождает аналитическую полугруппу. Согласно лемме 3.1 оператор $-\mathscr{A}$ является генератором аналитической полугруппы. В силу ограниченности оператора $C$ относительно $\mathscr{A}$ с относительной границей 0 справедливо следствие 2.5 [13; гл. 9], которое утверждает, что в этом случае оператор $-\mathscr{L}=-(\mathscr{A}+C)$ является генератором аналитической полугруппы $\left\{T_{t}\right\}_{t \geqslant 0}$, и имеет место оценка $\left\|T_{t}\right\| \leqslant M e^{\beta t}$.

$$
\text { Функция } u_{1}(t)=\int_{0}^{t} T_{t-s} f(s) d s \text { является сильным решением задачи }(5.1),(5.2)
$$

при $\varphi=0$. Оператор $-\mathscr{L}-\beta I$ является генератором ограниченной полугруппы $\widetilde{T}_{t}$, связанной с $T_{t}$ соотношением $\widetilde{T}_{t}=e^{-\beta t} T_{t}$. Функция $u_{2}(t)=e^{\beta t} \widetilde{T}_{t} \varphi$ будет сильньм решением задачи $(5.1),(5.2)$ при $f=0$ тогда и только тогда, когда

$$
\int_{0}^{T}\left\|e^{\beta t} \widetilde{T}_{t} \varphi\right\|_{H}^{2} d t \leqslant c_{3} \int_{0}^{T}\left\|\widetilde{T}_{t} \varphi\right\|_{H}^{2} d t<\infty
$$


В силу теоремы 1.14 .5 [14; гл. 1] последнее неравенство эквивалентно включению $\varphi \in[\mathscr{D}(\mathscr{L}+\beta I) ; H]_{1 / 2}$. Но поскольку $\mathscr{D}(\mathscr{L}+\beta I)=\mathscr{D}(\mathscr{A})$, то в силу теоремы 4.1 имеем $[\mathscr{D}(\mathscr{L}+\beta I) ; H]_{1 / 2}=V$.

Следовательно, $u=u_{1}+u_{2}$ будет сильным решением задачи (5.1), (5.2) тогда и только тогда, когда $\varphi \in V$.

Нам понадобится одна лемма о вложении интерполяционных пространств. Рассмотрим гильбертово пространство $\mathrm{H}_{2}$, относительного которого будем предполагать, что вложение $H_{2} \subset H$ плотно и непрерывно.

Лемма 5.1. Предположим, что пространство $\mathrm{H}_{2}$ непрерывно вложено в пространство $H_{1}$, тогда имеет место непрерывное влохсение $\left[H_{2} ; H\right]_{1 / 2} \subset$ $\left[H_{1} ; H\right]_{1 / 2}$.

ДокАЗАТЕльство. Для любых $t>0$ и $\psi \in H$ определим функционал

$$
K\left(t, \psi ; H_{1}, H\right)=\inf _{\substack{\psi_{0}+\psi_{1}=\psi \\ \psi_{0} \in H_{1} \\ \psi_{1} \in H}}\left(\left\|\psi_{0}\right\|_{H_{1}}+t\left\|\psi_{1}\right\|_{H}\right)
$$

В силу теоремы $15.1\left[12 ;\right.$ гл. 1] имеет место равенство $\left[H_{1} ; H\right]_{1 / 2}=\{\psi \in H$ : $\left.\int_{0}^{\infty} t^{-2} K^{2}\left(t, \psi ; H_{1}, H\right) d t<\infty\right\}$. В силу вложения $H_{2} \subset H_{1}$ имеет место оценка функции $K\left(t, \varphi ; H_{1}, H\right)$ при $t>0$

$$
K\left(t, \varphi ; H_{1}, H\right) \leqslant K\left(t, \varphi ; H_{2}, H\right)
$$

Поэтому если $t^{-1} K\left(t, \varphi ; H_{2}, H\right) \in L_{2}(0, \infty)$, то и $t^{-1} K\left(t, \varphi ; H_{1}, H\right) \in L_{2}(0, \infty)$. Следовательно, $\left[H_{2} ; H\right]_{1 / 2} \subset\left[H_{1} ; H\right]_{1 / 2}$.

Рассмотрим в качестве примера уравнение теплопроводности. Необходимые и достаточные условия сильной разрешимости первой смешанной задачи для такого уравнения изучались в работах [16]-[18].

Пусть $Q \subset \mathbb{R}^{n}$ - ограниченная область с границей $\partial Q$, удовлетворяющей условию Липшица. Через $Q_{T}$ обозначим ограниченньй цилиндр $Q_{T}=Q \times(0, T)$. Мы будем обозначать через $W_{2}^{k}(Q)$ пространство Соболева комплекснозначных функций из $L_{2}(Q)$, имеющих все обобшенные производные вплоть до $k$-го порядка из $L_{2}(Q)$, с нормой

$$
\|u\|_{W_{2}^{k}(Q)}=\left(\sum_{|\alpha| \leqslant k} \int_{Q}\left|\mathscr{D}^{\alpha} u(x)\right|^{2} d x\right)^{1 / 2}
$$

Через $\stackrel{\circ}{W}_{2}^{k}(Q)$ обозначим замькание множества финитных бесконечно дифференцируемых функций $C_{0}^{\infty}(Q)$ в $W_{2}^{k}(Q)$, а через $W_{2}^{-k}(Q)$ обозначим пространство, сопряженное к $\stackrel{\circ}{W} \underset{2}{k}(Q)$.

Рассмотрим первую смешанную задачу для уравнения теплопроводности

$$
u_{t}(x, t)-\Delta u(x, t)=f(x, t), \quad(x, t) \in Q_{T},
$$


с краевым условием

$$
\left.u\right|_{\partial Q \times(0, T)}=0
$$

и начальньм условием

$$
\left.u\right|_{t=0}=\varphi(x), \quad x \in Q .
$$

В качестве пространства $H$ возьмем пространство $L_{2}(Q)$, за пространство $V$ примем пространство $\stackrel{\circ}{W} \frac{1}{2}(Q)$, соответственно $V^{\prime}=W_{2}^{-1}(Q)$. Оператор $\mathrm{A}: \stackrel{\circ}{W} \underset{2}{1}(Q) \rightarrow$ $W_{2}^{-1}(Q)$ определим по формуле $\mathrm{A} u=-\Delta u$, где производные понимаются в смысле обобщенные производных.

Оператор А будет $\stackrel{\circ}{W} \underset{2}{1}(Q)$-коэрцитивным. Действительно, для любой $u \in C_{0}^{\infty}(Q)$ имеем

$$
\operatorname{Re}\langle\operatorname{A} u, u\rangle=-\operatorname{Re}(\Delta u, u)_{L_{2}(Q)}=\|\nabla u\|_{L_{2}(Q)}^{2} \geqslant c_{1}\|u\|_{W_{2}^{1}(Q)}^{2} .
$$

Задачу (5.3)- (5.5) можно переформулировать как задачу (5.1), (5.2).

Очевидно, $\stackrel{\circ}{W} \underset{2}{2}(Q)$ непрерывно вложено в $\mathscr{D}(\mathscr{A})$, где $\mathscr{A}$ - неограниченный оператор, построенный по оператору А согласно $\S 2$. В силу теоремы $11.6[12 ;$ гл. 1] имеет место равенство

$$
\left[\stackrel{\circ}{W}_{2}^{2}(Q) ; L_{2}(Q)\right]_{1 / 2}=\stackrel{\circ}{W}{ }_{2}^{1}(Q)
$$

Следовательно, согласно лемме $5.1 \stackrel{\circ}{\mathrm{W}} \underset{2}{1}(Q) \subset\left[\mathscr{D}(\mathscr{A}) ; L_{2}(Q)\right]_{1 / 2}$ и это вложение непрерывно. В силу теоремы 5.1 мы имеем следующий результат. Задача (5.3)-(5.5) имеет единственное сильное решение тогда и только тогда, когда $\varphi \in \stackrel{\circ}{W} \underset{2}{1}(Q)$.

Аналогично можно исследовать первую краевую задачу и для параболического уравнения с сильно эллиптическим оператором $2 m$-го порядка.

ЗАмечАниЕ 5.1. Теорема 11.6 [12; гл. 1] сформулирована для областей, имеющих гладкие границы. Однако используя теорему о продолжении функций для липшицевых областей [19; $\S 3$, гл. 6, теорема 5], равенство (5.6) можно доказать и для областей, имеющих липшицевы границы.

Рассмотрим применение теоремы 5.1 для параболических функционально-дифференциальных уравнений. Как уже отмечалось, такие уравнения обладают рядом принципиально новых свойств, например, гладкость сильных решений может нарушаться внутри цилиндрической области. Тем не менее, оказывается, что необходимое и достаточное условие сильной разрешимости для параболических функционально-дифференциальных уравнений совпадает с критерием сильной разрешимости уравнения теплопроводности.

Рассмотрим ограниченный оператор $A_{B}: \stackrel{\circ}{W} \underset{2}{1}(Q) \rightarrow W_{2}^{-1}(Q)$, действующий по формуле $A_{B} u=-\operatorname{div}(B \nabla u)$, где $B: L_{2}^{n}(Q) \rightarrow L_{2}^{n}(Q)$ - ограниченный оператор. Мы обозначили

$$
L_{2}^{n}(Q)=\prod_{k=1}^{n} L_{2}(Q), \quad \stackrel{\circ}{W}_{2}^{1, n}(Q)=\prod_{k=1}^{n} \stackrel{\circ}{W_{2}^{1}}(Q), \quad W_{2}^{1, n}(Q)=\prod_{k=1}^{n} W_{2}^{1}(Q) .
$$

Относительно оператора $B$ будем предполагать, что выполнены следующие условия. 
УСловиЕ 5.1. Оператор $B$ ограниченно отображает пространство $\stackrel{\circ}{W_{2}^{1, n}}(Q)$ в пространство $W_{2}^{1, n}(Q)$.

УС ловиЕ 5.2. Оператор $A_{B}$ является $\stackrel{\circ}{W} \underset{2}{1}(Q)$-коэрцитивньп.

Через $\mathscr{A}_{B}$ обозначим неограниченный оператор, построенный согласно $\S 2$.

Рассмотрим первую смешанную задачу для параболического операторно-дифференциального уравнения

$$
u_{t}(x, t)+\mathscr{A}_{B} u(x, t)+C u(x, t)=f(x, t), \quad(x, t) \in Q_{T}
$$

с краевым условием

$$
\left.u\right|_{\partial Q \times(0, T)}=0
$$

и начальньм условием

$$
\left.u\right|_{t=0}=\varphi(x), \quad x \in Q .
$$

Задачу (5.7)-(5.9) можно рассматривать как задачу (5.1), (5.2).

Заметим, что из выполнения условия 5.2 для оператора $B$ следует вьполнение условия 5.2 и для оператора $B^{*}$. Сопряженным к оператору $\mathscr{A}_{B}$ будет оператор $\mathscr{A}_{\mathscr{B}} *$. Действительно, для любых $u, v \in C_{0}^{\infty}(Q)$

$$
\left(\mathscr{A}_{B} u, v\right)_{L_{2}(Q)}=-(B \nabla u, \nabla v)_{L_{2}^{n}(Q)}=\left(u, \mathscr{A}_{\mathscr{B}} * v\right)_{L_{2}(Q)}
$$

Поскольку $C_{0}^{\infty}(Q)$ всюду плотно в $\stackrel{\circ}{W} \underset{2}{1}(Q)$, тождества $(5.10)$ справедливы для любых $u \in \mathscr{D}\left(\mathscr{A}_{B}\right), v \in \mathscr{D}\left(\mathscr{A}_{\mathscr{B}} *\right)$. Следовательно, $\mathscr{A}_{\mathscr{B}} * \subset\left(\mathscr{A}_{B}\right)^{*}$ и $\mathscr{A}_{B} \subset\left(\mathscr{A}_{\mathscr{B}}\right)^{*}$. Однако в силу $\stackrel{\circ}{W}_{2}^{1}(Q)$-коэрцитивности операторов $\mathscr{A}_{B}$ и $\mathscr{A}_{\mathscr{B}} * 0 \notin \sigma\left(\mathscr{A}_{B}\right) \cup \sigma\left(\mathscr{A}_{\mathscr{B}} *\right)$, тогда по лемме $13\left[20 ; \S 6\right.$, гл. 14] о сопряженных операторах $\left(\mathscr{A}_{B}\right)^{*}=\mathscr{A}_{\mathscr{B}^{*}}$.

Поскольку оператор $B$ ограниченно отображает $\stackrel{\circ}{W}_{2}^{1, n}(Q)$ в $W_{2}^{1, n}(Q)$, то имеет место непрерывное вложение $\stackrel{\circ}{W} \underset{2}{2}(Q) \subset \mathscr{D}\left(\mathscr{A}_{B}\right)$. Соответственно $\stackrel{\circ}{W}{ }_{2}^{2}(Q)$ непрерывно вложено в $\mathscr{D}\left(\mathscr{A}_{\mathscr{B}} *\right)$. В силу леммы 5.1 имеют место непрерывные вложения $\stackrel{\circ}{W}_{2}^{1}(Q) \subset\left[\mathscr{D}\left(\mathscr{A}_{B}\right) ; L_{2}(Q)\right]_{1 / 2}$ и $\stackrel{\circ}{W}_{2}^{1}(Q) \subset\left[\mathscr{D}\left(\mathscr{A}_{\mathscr{B}} *\right) ; L_{2}(Q)\right]_{1 / 2}$.

Таким образом, для задачи (5.7)-(5.9) выполнены условия теоремы 5.1 , и мы имеем следующий результат.

ТЕОРема 5.2. Пусть оператор В удовлетворяет условиям 5.1 и 5.2, а оператор $B^{*}$ удовлетворяет условию 5.1 .

Тогда для любого $f \in L_{2}\left(0, T ; L_{2}(Q)\right)$ задача $(5.7)-(5.9)$ имеет единственное сильное решение тогда и только тогда, когда $\varphi \in \stackrel{\circ}{W} \underset{2}{1}(Q)$.

Приведем примеры операторов $B$, для которых выполнены условия 5.1, 5.2. Покажем, что для важных классов функционально-дифференциальных уравнений применима теорема 5.2.

Сделаем дополнительные предположения относительно области $Q$. Пусть гранища области $Q$ представляется следующим объединением:

$$
\partial Q=\bigcup_{i} \overline{M_{i}}, \quad i=1, \ldots, N_{0}
$$


где $M_{i}-(n-1)$-мерные многообразия класса $C^{\infty}$, которые являются открытьми и связными в топологии $\partial Q$. Пусть в окрестности каждой точки $g \in \partial Q \backslash \bigcup_{i} M_{i}$ область $Q$ диффеоморфна $n$-мерному двугранному углу, если $n \geqslant 3$, и плоскому углу, если $n=2$.

Введем ограниченные разностные операторы $R_{i j}: L_{2}\left(\mathbb{R}^{n}\right) \rightarrow L_{2}\left(\mathbb{R}^{n}\right)$ и $R_{i j Q}: L_{2}(Q) \rightarrow L_{2}(Q)$ по формулам

$$
R_{i j} u(x)=\sum_{h \in M} a_{i j h}(x) u(x+h), \quad R_{i j Q} v=P_{Q} R_{i j} I_{Q} v .
$$

Здесь $M \subset \mathbb{R}^{n}$ - конечное множество векторов с целочисленными координатами, $a_{i j h} \in C^{\infty}\left(\mathbb{R}^{n}\right)$ - комплекснозначные функции, $I_{Q}$ - оператор продолжения функций из $L_{2}(Q)$ нулем в $\mathbb{R}^{n} \backslash Q, P_{Q}$ - оператор сужения функций из $L_{2}\left(\mathbb{R}^{n}\right)$ на $Q$.

В качестве оператора $B$ возьмем оператор $R: L_{2}^{n}(Q) \rightarrow L_{2}^{n}(Q)$, введенньй по формуле

$$
(R u)_{i}=\sum_{j=1}^{n} R_{i j Q} u_{j}, \quad i=1,2, \ldots, n,
$$

где $u=\left(u_{1}, \ldots, u_{n}\right)^{T}$.

Будем рассматривать дифференциально-разностньй оператор $A_{R}$, заданный формулой $A_{R}=-\operatorname{div}(R \nabla u)$. Соответственно введем неограниченный оператор $\mathscr{A}_{R}: \mathscr{D}\left(\mathscr{A}_{R}\right) \subset L_{2}(Q) \rightarrow L_{2}(Q)$.

Для того чтобы сформулировать условия $\stackrel{\circ}{W} \frac{1}{2}(Q)$-коэрцитивности оператора $A_{R}$, следуя [9], введем некоторые вспомогательные обозначения. Обозначим через $G$ аддитивную абелеву группу, порожденную множеством $M$, а через $Q_{r}-$ открытые связные компоненты множества $Q \backslash\left(\bigcup_{h \in G}(\partial Q+h)\right)$.

ОПРЕДЕЛЕНИЕ 5.2. Множества $Q_{r}$ мы будем называть подобластями а совокупность $\mathscr{R}$ всевозможных подобластей $Q_{r}$ - разбиением множества $Q$.

Разбиение $\mathscr{R}$ естественным образом распадается на непересекающиеся классы: будем считать, что подобласти $Q_{r_{1}}, Q_{r_{2}} \in \mathscr{R}$ принадлежат одному классу, если существует $h \in G$ такое, что $Q_{r_{2}}=Q_{r_{1}}+h$. Обозначим подобласти $Q_{r}$ через $Q_{s l}$, где $s$-номер класса, $s=1,2, \ldots$, a $l$-порядковьй номер подобласти в $s$-ом классе. В силу ограниченности области $Q$ каждый класс состоит из конечного числа $N=$ $N(s)$ подобластей $Q_{s l}$ и $N(s) \leqslant([\operatorname{diam} Q]+1)^{n}$.

Для того чтобы сформулировать необходимые условия $\stackrel{\circ}{W} \underset{2}{1}(Q)$-коэрцитивности в алгебраической форме, мы введем матрицы $R_{i j s}(x), x \in \bar{Q}_{s 1}$, порядка $N(s) \times$ $N(s)$ с элементами

$$
r_{k l}^{i j s}(x)= \begin{cases}a_{i j h}\left(x+h_{s k}\right), & h=h_{s l}-h_{s k} \in M \\ 0, & h_{s l}-h_{s k} \notin M .\end{cases}
$$

В силу теоремы 9.1 [9; гл. 2$]$ если оператор $A_{R}$ является $\stackrel{\circ}{W} \stackrel{1}{2}(Q)$-коэрцитивным, то для всех $s=1,2, \ldots, x \in \bar{Q}_{s 1}$ и $0 \neq \xi \in \mathbb{R}^{n}$ матрицы

$$
\sum_{i, j=1}^{n}\left(R_{i j s}(x)+R_{i j s}^{*}(x)\right) \xi_{i} \xi_{j}
$$

положительно определены. 
Пусть теперь $x \in \bar{Q}_{s 1}$ - произвольная точка. Рассмотрим все точки $x^{l} \in \bar{Q}$ такие, что $x^{l}-x \in G$. Поскольку область $Q$ ограниченная, множество $\left\{x^{l}\right\}$ состоит из конечного числа точек $I=I(s, x), I \geqslant N(s)$. Перенумеруем точки $x^{l}$ так, что $x^{l}=x+h_{s l}$ для $l=1, \ldots, N=N(s), x^{1}=x$, где $h_{s l}$ удовлетворяет условию $Q_{s l}=Q_{s 1}+h_{s l}$.

Введем матрицы $A_{i j s}(x)$ порядка $I \times I$ с элементами $a_{l k}^{i j s}(x)$ по формуле

$$
a_{l k}^{i j s}(x)= \begin{cases}a_{i j h}\left(x^{l}\right), & h=x^{k}-x^{l} \in M \\ 0, & x^{k}-x^{l} \notin M .\end{cases}
$$

В силу теоремы 9.2 [9; гл. 2] если для всех $s=1,2, \ldots, x \in \bar{Q}_{s 1}$ и $0 \neq \xi \in \mathbb{R}^{n}$ матрицы

$$
\sum_{i, j=1}^{n}\left(A_{i j s}(x)+A_{i j s}^{*}(x)\right) \xi_{i} \xi_{j}
$$

положительно определены, то оператор $A_{R}$ является $\stackrel{\circ}{W} \frac{1}{2}(Q)$-коэрцитивным.

Очевидно, если $I=N$, то матрица $R_{i j s}(x)$ равна матрице $A_{i j s}(x)$. Если $N<I$, то матрица $R_{i j s}(x)$ получается из матрицы $A_{i j s}$ вычеркиванием последних $I-N$ строк и столбцов.

Рассмотрим параболическое дифференциально-разностное уравнение

$$
u_{t}(x, t)+\mathscr{A}_{R} u(x, t)+C u(x, t)=f(x, t), \quad(x, t) \in Q_{T},
$$

с краевым условием

$$
\left.u\right|_{\partial Q \times(0, T)}=0
$$

и начальньгм условием

$$
\left.u\right|_{t=0}=\varphi(x), \quad x \in Q
$$

ТЕОРЕМА 5.3. Пусть выполнено условие $\stackrel{\circ}{W} \underset{2}{1}(Q)$-коэрцитивности для опеpamopa $\mathscr{A}_{R}$.

Тогда задача (5.11)-(5.13) имеет единственное сильное решение тогда и только тогда, когда $\varphi \in \stackrel{\circ}{W} \underset{2}{1}(Q)$.

ДокАЗАТЕльство. Покажем, что оператор $R$ удовлетворяет условиям 5.1 и 5.2. Действительно, в силу леммы 8.13 [9; гл. 2] оператор $R$ непрерьвно отображает $\stackrel{\circ}{W} \underset{2}{1, n}(Q)$ в $W_{2}^{1, n}(Q)$. А по условию теоремы оператор $\mathscr{A}_{R}$ является $\stackrel{\circ}{W} \underset{2}{1}(Q)$-коэрцитивным. Аналогично, $R^{*}$ удовлетворяет условию 5.1.

Таким образом, к задаче (5.11)-(5.13) применима теорема 5.2.

ПримеР 5.1. Рассмотрим задачу (5.11)-(5.13), предполагая, что $Q=\left(0, \frac{4}{3}\right) \times$ $\left(0, \frac{4}{3}\right), A_{R}=-\operatorname{div}\left(R_{Q} \nabla u\right), R_{Q}=P_{Q} R I_{Q}, R u(x)=u(x)+a u\left(x_{1}+1, x_{2}+1\right)+$ $a u\left(x_{1}-1, x_{2}-1\right), 0<a<1$. Очевидно, разбиение $\mathscr{R}$ области $Q$ состоит из двух классов подобластей:

1) $Q_{11}=\left(0, \frac{1}{3}\right) \times\left(0, \frac{1}{3}\right), Q_{12}=\left(1, \frac{4}{3}\right) \times\left(1, \frac{4}{3}\right)$;

2) $Q_{21}=Q \backslash\left(\bar{Q}_{11} \cup \bar{Q}_{12}\right)$. 
Введем множество $\mathscr{K} \subset \partial Q$, состоящее из из четырех точек:

$$
g^{1}=\left(\frac{1}{3}, 0\right), \quad g^{2}=\left(\frac{4}{3}, 1\right), \quad g^{3}=\left(0, \frac{1}{3}\right), \quad g^{4}=\left(1, \frac{4}{3}\right)
$$

Матрицы $A_{s}(x), x \in \bar{Q}_{s 1}, s=1,2$, имеют вид

$$
\begin{gathered}
A_{1}(x)=\left(\begin{array}{ll}
1 & a \\
a & 1
\end{array}\right), \quad x \in \bar{Q}_{11}, \\
A_{2}(x)=\left(\begin{array}{ll}
1 & a \\
a & 1
\end{array}\right), \quad x \in \bar{Q}_{21} \cap \mathscr{K}, \quad A_{2}(x)=(1), \quad x \in \bar{Q}_{21} \backslash \mathscr{K} .
\end{gathered}
$$

Таким образом, матрицы $A_{s}(x)\left(\xi_{1}^{2}+\xi_{2}^{2}\right), x \in \bar{Q}_{s 1}, s=1,2$, положительно определены. Следовательно, оператор $A_{R}$ является $\stackrel{\circ}{W} \underset{2}{1}(Q)$-коэрцитивным.

Согласно теореме 5.3 задача (5.11)-(5.13) имеет единственное сильное решение тогда и только тогда, когда $\varphi \in \stackrel{\circ}{W} \underset{2}{1}(Q)$.

Однако в [9] доказано, что $\mathscr{D}\left(\mathscr{A}_{R}\right) \not \subset W_{2}^{2}(Q)$. Используя этот результат, в работе [7] было показано, что имеет место нарушение гладкости сильных решений на границе соседних подобластей $Q_{s_{1} l_{1}} \times(0, T), Q_{s_{2} l_{2}} \times(0, T)$ и вблизи множества $\mathscr{K} \times(0, T)$. В этом примере область определения $\mathscr{D}\left(\mathscr{A}_{R}\right)$ не может быть описана в терминах пространств Соболева. Тем не менее, используя подходы, преложенные в настоящей статье, мы имеем описание пространства начальных данных в виде пространства Соболева.

Введем теперь операторы растяжения и сжатия $T_{i j}: L_{2}\left(\mathbb{R}^{n}\right) \rightarrow L_{2}\left(\mathbb{R}^{n}\right)$ и $T_{i j Q}: L_{2}(Q) \rightarrow L_{2}(Q)$ по формулам

$$
T_{i j} u(x)=\sum_{l \in N} a_{i j l} u\left(q^{-l} x\right), \quad T_{i j Q} v=P_{Q} T_{i j} I_{Q} v
$$

где $N \subset \mathbb{N}$ - конечное множество натуральных чисел, $a_{i j l} \in \mathbb{C}, q>1$, операторы $I_{Q}$ и $P_{Q}$ такие же, как в определении разностных операторов.

Введем оператор Т: $L_{2}^{n}(Q) \rightarrow L_{2}^{n}(Q)$ по формуле

$$
(\mathrm{T} u)_{i}=\sum_{j=1}^{n} T_{i j Q} u_{j}, \quad i=1,2, \ldots, n,
$$

где $u=\left(u_{1}, \ldots, u_{n}\right)^{T}$. Возьмем в качестве оператора $B$ оператор Т и рассмотрим функционально-дифференциальный оператор $A_{\mathrm{T}}$ с растяжением и сжатием аргументов. Соответственно введем неограниченньй оператор $\mathscr{A}_{\mathrm{T}}: \mathscr{D}\left(\mathscr{A}_{\mathrm{T}}\right) \subset L_{2}(Q) \rightarrow$ $L_{2}(Q)$.

Обозначим $t_{i j}(\lambda)=\sum_{l \in N} a_{i j l} \lambda^{l}$. В силу теоремы 1 [21] условие

$$
\sum_{i, j=1}^{n} t_{i j}(\lambda) \xi_{i} \xi_{j}>0, \quad \lambda \in \mathbb{C}, \quad|\lambda|=q^{n / 2}, \quad 0 \neq \xi \in \mathbb{R}^{n},
$$

является достаточным для $\stackrel{\circ}{W} \underset{2}{1}(Q)$-коэрцитивности оператора $\mathscr{A}_{\mathrm{T}}$. Если дополнительно предположить, что область $Q$ удовлетворяет условию

$$
\bar{Q} \subset q Q,
$$


то в силу теоремы 2 [21] условие (5.14) является и необходимым для $\stackrel{\circ}{W} \underset{2}{1}(Q)$-коэрцитивности оператора $\mathscr{A}_{\mathrm{T}}$.

Рассмотрим параболическое функционально-дифференциальное уравнение с растяжением и сжатием аргументов

$$
u_{t}(x, t)+\mathscr{A}_{\mathrm{T}} u(x, t)+C u(x, t)=f(x, t), \quad(x, t) \in Q_{T},
$$

с краевым условием

$$
\left.u\right|_{\partial Q \times(0, T)}=0
$$

и начальньм условием

$$
\left.u\right|_{t=0}=\varphi(x), \quad x \in Q
$$

ТЕОРема 5.4. Пусть для оператора Т выполнено условие (5.14). Тогда задача (5.15)-(5.17) имеет единственное сильное решение тогда и только тогда, когда $\varphi \in \stackrel{\circ}{W} \underset{2}{1}(Q)$.

ДоказАТЕльство. Покажем, что операторы Т, T* удовлетворяют условию 5.1. Легко видеть, что операторы $\mathrm{T}, \mathrm{T}^{*}$ ограниченно отображают $\stackrel{\circ}{W} \underset{2}{1, n}(Q)$ в $W_{2}^{1, n}(Q)$. По условию теоремы $\mathscr{A}_{\mathrm{T}}$ и $\mathscr{A}_{\mathrm{T}} *$ будут $\stackrel{\circ}{W} \underset{2}{1}(Q)$-коэрцитивньми.

Таким образом, к задаче (5.15)-(5.17) применима теорема 5.2.

ПримеР 5.2. Пусть $Q=\{|x|<1\} \subset \mathbb{R}^{n}$. Рассмотрим задачу

$$
\begin{gathered}
u_{t}(x, t)-\Delta\left(u(x, t)+a_{1} u\left(q^{-1} x, t\right)\right)=f(x, t), \quad(x, t) \in Q_{T}, \\
\left.u\right|_{\partial Q \times(0, T)}=0, \\
\left.u\right|_{t=0}=\varphi(x), \quad x \in Q,
\end{gathered}
$$

где $q>1, a_{1} \in \mathbb{R}$.

В данном примере условие (5.14) означает $\left|a_{1}\right| \leqslant q^{1-n / 2}$. По теореме 5.4 задача (5.18)-(5.20) имеет сильное решение тогда и только тогда, когда $\varphi \in \stackrel{\circ}{W} \underset{2}{1}(Q)$.

Автор выражает глубокую благодарность А. Л. Скубачевскому за постоянное внимание к работе и ряд важных замечаний.

\section{Список литературы}

1. Ashyralyev A., Sobolevskii P. E. Well-posedness of parabolic difference equations. Basel: Birkhäuser, 1994.

2. Pazy A. Semigroups of linear operators and applications to partial differential equations. New York: Springer-Verlag, 1983.

3. Kato T. Fractional powers of dissipative operators // J. Math. Soc. Japan. 1961. V. 13. P. 246-274. 
4. McIntosh $A$. On the comparability of $A^{1 / 2}$ and $A^{* 1 / 2} / /$ Proc. Amer. Math. Soc. 1972 . V. 32. P. 430-434.

5. Auscher P., Hofmann S., McIntosh A., Tchamitchian P. The Kato square root problem for higher order elliptic operators and systems on $\mathbb{R}^{n} / /$ J. Evolution Equations. 2001. V. 1. № 4. P. 361-385.

6. Kato T., McLeod J.B. The functional differential equation // Bull. Amer. Math. Soc. 1971. V. 77. P. 891-937.

7. Скубачевский A. Л., Шамин Р. В. Первая смешанная задача для параболического дифференциально-разностного уравнения // Матем. заметки. 1999. Т. 66. № 1. С. 145-153.

8. Skubachevskii A. L., Shamin $R$. V. The mixed boundary value problem for parabolic differential-difference equation // Funct. Differ. Equ. 2001. V. 8. № 3-4. P. 407-424.

9. Skubachevskii A.L. Elliptic functional differential equations and applications. Basel: Birkhäuser, 1997.

10. Скубачевский А. Л., Шамин Р. В. Параболические дифференциально-разностные уравнения второго порядка // Докл. РАН. 2001. Т. 379. № 5. С. 735-738.

11. Шамин Р. В. Пространства начальных данных для параболических функционально-дифференциальных уравнений // Матем. заметки. 2002. Т. 71. № 4. С. 636-640.

12. Лионс ЖК.-Л., Мадженес Э. Неоднородные граничные задачи и их приложения. М.: Мир, 1971.

13. Като Т. Теория возмущений линейных операторов. М.: Мир, 1972.

14. Трибель $X$. Теория интерполяции, функциональные пространства, дифференциальные операторы. М.: Мир, 1980.

15. Красносельский М.А., Забрейко П. П., Пустыльник Е. И., Соболевский П. Е. Интегральные операторы в пространствах суммируемых функций. М.: Наука, 1966.

16. Слободецкий Л. Н. Обобщенные пространства С. Л. Соболева и их приложения к краевым задачам для дифференциальных уравнений в частных производных // Уч. записки Лен. пед. инст. им. А.И. Герцена. 1958. Т. 197. С. 54-112.

17. Ладыжсенская O.A. О нестационарных операторных уравнениях и их приложениях к линейньм задачам математической физики // Матем. сб. 1958. Т. 45. № 2. С. 123-158.

18. Ладызсенская $О$. А. О решении нестационарных операторных уравнений // Матем. сб. 1956. T. 39. № 4. C. 491-524.

19. Стейн И. Сингулярные интегралы и дифференциальные свойства функций. М.: Мир, 1973.

20. Данфорд Н., Швари Джс. Линейные операторы. Т. 2. М.: Мир, 1966.

21. Россовский Л. Е. Коэрцитивность функционально-дифференциальных уравнений // Матем. заметки. 1996. Т. 59. № 1. С. 103-113.

Московский авиационный институт

Поступила в редакцию

E-mail: rwsh@yandex.ru 